\title{
Avaliação da microdureza e da rugosidade de compósitos resinosos de uso direto e indireto
}

\author{
Assessing microhardness and roughness of composite resins of direct \\ and indirect use
}

lana Sá de Oliveira*

Vanessa Fontenele Marques ${ }^{* *}$

Denise Sá Maia Casselli ${ }^{* * *}$

\section{Resumo}

Objetivo: avaliar rugosidade superficial por meio de perfilometria de contato mecânico bem como a resistência à microdureza Knoop em resinas fotoativadas de forma convencional ou com sistemas de polimerização adicional. Materiais e método: 82 corpos de prova foram distribuídos nos seguintes grupos: SI: Solidex ${ }^{\circledR}$, Z1D: Z100 ${ }^{\circledR}, Z 35 D: Z 350^{\circledR}$, OD: Opallis ${ }^{\circledR}, Z 11: Z 100^{\circledR}$, Z35I: Z350 ${ }^{\circledR}$ e OI: Opallis ${ }^{\circledR}$. Os incrementos de resina foram fotopolimerizados por 40 segundos cada. O grupo contendo a resina de uso indireto Solidex ${ }^{\circledR}(\mathrm{SI})$ e os grupos Z1I, Z35I e OI contendo resinas de uso direto avaliadas como uso indireto tiveram fotopolimerização por 40 segundos mais 7 minutos adicionais com EDG- LUX. As amostras foram armazenadas em água destilada a $37^{\circ} \mathrm{C}$ por 24 horas. Em seguida foi realizado o ensaio de rugosidade e posteriormente o de microdureza superficial. As médias coletadas foram analisadas pelo teste não paramétrico de Kruskal-Wallis e o de comparação múltipla de Dunn. Resultados: a resina indicada para uso direto $Z 100^{\circledR}$ obteve os maiores valores de microdureza, independente da presença de fotoativação adicional. Já a resina indicada para uso indireto Solidex, obteve os menores valores de microdureza. Os valores de rugosidade não apresentaram diferenças estatísticas entre os materiais analisados. Conclusão: resinas de uso direto com polimerização adicional podem ser bem utilizadas na forma indireta.

Palavras-chave: Resinas Compostas. Microdureza. Rugosidade.

\section{Introdução}

As resinas compostas são materiais estéticos que vem ganhando grande aplicabilidade na Odontologia atual, tanto nas restaurações de dentes anteriores como posteriores ${ }^{1}$. Um marco importante foi o desenvolvimento de resinas compostas fotoativadas para procedimentos diretos ${ }^{2}$ em que a longevidade clínica dessas restaurações está vinculada de maneira indelével com a adoção de adequada fotoativação ${ }^{3}$.

É evidente que as resinas compostas foram desenvolvidas com o objetivo de reparar as perdas de material dentário por apresentarem menor desgaste, maior facilidade de manuseio e por possuírem uma grande gama de cores. Tornam-se praticamente imperceptíveis após a sua aplicação, possibilitando alcançar semelhança com a cor dos dentes humanos ${ }^{4}$.

Com o desenvolvimento do condicionamento ácido, as resinas compostas passaram por diversas modificações em sua composição química com o propósito de melhorar suas propriedades mecânicas e, consequentemente, seu desempenho clínico ${ }^{5-7}$. A composição do material exerce grande influência sobre as propriedades físicas e mecânicas dos compósitos, da mesma maneira que o método de foto- 
ativação. Tanto o tamanho, formato, composição e distribuição das partículas, como principalmente o tipo de matriz orgânica utilizada nos compósitos influencia de forma direta as propriedades físicas desses materiais ${ }^{8,9}$.

A variação no tamanho e formato das partículas inorgânicas que as compõem tem proporcionado melhora na lisura superficial e menor aderência de biofilme, melhores propriedades ópticas que se refletem em qualidade estética superior e maior durabilidade das restaurações ${ }^{10}$.

As resinas compostas laboratoriais são modificações de sistemas para uso direto, com alterações de formas de ativação e polimerização. Essas resinas compostas passam por uma polimerização mais efetiva, resultando em maior grau de conversão de polimerização. Uma melhoria geral das propriedades físicas pode ser esperada ${ }^{11}$. As resinas compostas indiretas, apesar das vantagens, são aproximadamente duas vezes mais caras do que os compósitos diretos ${ }^{12,13}$.

Apesar de todos os avanços observados no desenvolvimento das resinas compostas, percebe-se que esses materiais ainda apresentam algumas li- mitações como a contração de polimerização, o coeficiente de expansão térmica linear distinta das estruturas dentárias e do grau de conversão dos monômeros ${ }^{1,14}$.

O ensaio de microdureza Knoop avalia a resistência à penetração em um material. Em resinas compostas, ele avalia indiretamente a eficácia da polimerização e o grau de conversão de monômeros em polímeros ${ }^{1,14}$. Os resultados obtidos após a aplicação desse teste possibilitam analisar se houve ou não uma adequada conversão desses monômeros, a partir dos resultados maiores ou menores da microdureza avaliada ${ }^{15,16}$.

$\mathrm{O}$ presente estudo tem por objetivo avaliar e comparar a rugosidade superficial média por meio de perfilometria de contato mecânico e a resistência à microdureza Knoop de sete grupos de resinas fotoativadas direta ou indiretamente.

\section{Materiais e método}

Os materiais empregados na presente pesquisa e suas respectivas composições estão listados na Tabela 1.

Tabela 1- Materiais utilizados no estudo e respectivas composições

\begin{tabular}{|c|c|c|}
\hline Marca Comercial & Fabricante & Composição $^{1}$ \\
\hline $\begin{array}{l}\text { Solidex } \\
\text { (indicação: uso } \\
\text { indireto) }\end{array}$ & $\begin{array}{l}\text { Shofu } \\
\text { (Shofu, Kyoto, Kinki, Japan) }\end{array}$ & $\begin{array}{l}\text { Bis-GMA, UDMA, TEGMA } \\
53 \% \text { microcarga de cerâmica inorgânica; } \\
25 \% \text { co-polímeros com resina multifuncional; } \\
22 \% \text { resinas convencionais/fotoiniciadores }\end{array}$ \\
\hline $\begin{array}{l}\mathrm{Z100}{ }^{\circledR} \\
\text { (indicação: uso direto) }\end{array}$ & $\begin{array}{l}\text { 3M ESPE } \\
\text { (3M ESPE, Sumaré, São Paulo, } \\
\text { Brasil) }\end{array}$ & $\begin{array}{l}\text { Bis-GMA, TEGDMA } \\
\text { 71\% volume: Zircônia e Sílica }\end{array}$ \\
\hline $\begin{array}{l}\text { Z350 } \\
\text { (indicação: uso direto) }\end{array}$ & $\begin{array}{l}\text { 3M ESPE } \\
\text { (3M ESPE, Sumaré, São Paulo, } \\
\text { Brasil) }\end{array}$ & $\begin{array}{l}\text { Bis-GMA, Bis-EMA, UDMA, com pequenas quantidades de } \\
\text { TEGDMA } \\
63,3 \% \text { volume: zircônia/sílica }\end{array}$ \\
\hline $\begin{array}{l}\text { Opallis }^{\circledR} \\
\text { (indicação: uso direto) }\end{array}$ & $\begin{array}{l}\text { FGM } \\
\text { (FGM, Joinville, Santa Catarina, } \\
\text { Brasil) }\end{array}$ & $\begin{array}{l}\text { Bis-GMA, Bis-EMA, TEGDMA; } \\
58,0 \% \text { volume: vidro de Ba-Al silicato silanizado/ } \\
\text { nanopartículas de dióxido de silício }\end{array}$ \\
\hline
\end{tabular}

Os espécimes cilíndricos foram confeccionados utilizando matriz bipartida de teflon (4 $\mathrm{mm}$ de diâmetro por $2 \mathrm{~mm}$ de profundidade), por meio da inserção dos materiais. Uma lâmina de vidro foi utilizada com o objetivo de obter pastilhas de resina composta com base plana e polida. As resinas foram inseridas em dois incrementos, os quais foram polimerizados de acordo com os grupos experimentais. Foram confeccionados 82 corpos de prova, distribuídos em sete grupos experimentais, descritos na Tabela 2 .
Tabela 2 - Grupos experimentais

\begin{tabular}{l|l|c}
\hline \multicolumn{1}{c|}{ Grupos } & \multicolumn{1}{|c}{ Resina Composta } & $\begin{array}{c}\text { № de corpos } \\
\text { de prova }\end{array}$ \\
\hline SI & Solidex $^{\circledR}$ (ativação adicional) & 12 \\
Z1D & Z100 & 12 \\
Z35D & Z350 & \\
OD & Opallis & 12 \\
Z1I & Z100 $^{\circledR}$ (ativação adicional) & 14 \\
Z35I & Z350 $^{\circledR}$ (ativação adicional) & 8 \\
OI & Opallis $^{\circledR}$ (ativação adicional) & 12 \\
\hline
\end{tabular}


Nos grupos Z1D, Z35D e OD, os espécimes foram confeccionados pelo processo de fotopolimerização por 40 segundos para cada incremento. Já nos grupos SI, Z1I, Z35I, OI, os incrementos, além de serem fotopolimerizados de forma convencional também tiveram uma polimerização adicional com EDG-LUX (EDG, São Carlos, São Paulo, Brasil) durante 7 minutos. As amostras obtidas foram armazenadas em um recipiente com água destilada $\mathrm{e}$ mantidas a $37^{\circ} \mathrm{C}$ por 24 horas.

Utilizando-se uma embutidora metalográfica (Arotec SA, Cotia, SP, Brasil), os espécimes foram embutidos em resina acrílica. Após essa fase, as amostras foram sequencialmente lixadas com lixas de granulação e depois polidas com feltro umedecido com pasta diamantada.

Após o polimento das amostras, foi realizada a perfilometria com perfilômetro Hommel Tester T1000 (Jenoptik AG, Jena, Alemanha) tendo sido obtidas três leituras em cada bloco. As medidas foram obtidas em Ra (rugosidade superficial média) e a unidade de medida foi o micrômetro ( $\mu \mathrm{m})$.

A microdureza foi avaliada após o teste de rugosidade, pois esse experimento resulta em endentações, o que poderia interferir nas medidas da perfilometria dos corpos de prova. Foi utilizado um microdurômetro (Future Tech, Tóquio, Japão) com penetrador tipo Knoop, com carga estática de 25 gramas, por cinco segundos. A medida de maior diagonal da endentação foi anotada e convertida em valor de dureza Knoop (KHN) pela seguinte fórmula: $\mathrm{KHN}=14.229 \times \mathrm{P} / \mathrm{I}^{2}$, em que $\mathrm{P}=$ Carga aplicada (Carga 25g / 5s); I = Comprimento da maior diagonal da penetração $(\mu \mathrm{m})$. Foram realizadas dez leituras em cada amostra, de forma que a média dos valores obtidos em cada amostra foi utilizada na análise estatística.

Os dados coletados foram analisados estatisticamente pelo teste não paramétrico de Kruskal-Wallis e o de comparação múltipla de Dunm, procurando avaliar a ocorrência de possível diferença estatística ou não, entre os grupos experimentais do presente trabalho.

\section{Resultados}

Os dados de dureza apresentaram normalidade, mas não tiveram variância homogênea, impossibilitando o uso de teste paramétrico. Utilizou-se, assim, o teste de Kruskal-Wallis, que demonstrou efeito significativo de tratamento $(\mathrm{P}<0,001)$. O teste de Dunn foi usado para comparações múltiplas e os resultados estão descritos na Tabela 3 , em função do uso de teste não paramétrico.
Tabela 3 - Resultados para o teste de microdureza

\begin{tabular}{l|l}
\hline \multicolumn{1}{c|}{ Grupo } & \multicolumn{1}{c}{ Mediana } \\
\hline Z1D & $140,9 \mathrm{~A}$ \\
Z1I & $121,2 \mathrm{~A}$ \\
Z35I & $72,2 \mathrm{AB}$ \\
OD & $64,2 \mathrm{BC}$ \\
Z35D & $60,4 \mathrm{BC}$ \\
OI & $51,1 \mathrm{BC}$ \\
SI & $48,2 \mathrm{C}$ \\
\hline
\end{tabular}

Letras distintas indicam diferença estatística significante ao teste de Dunn $(\alpha=0,05)$

A resina indicada para uso direto Z100 (grupos Z1D e Z1D) obteve os maiores valores de microdureza, independente da presença de fotoativação adicional. Já a resina indicada para uso indireto Solidex (grupo SI) obteve os menores valores de microdureza. Os grupos Z35D; Z35I, OD e OI apresentaram valores de microdureza intermediários e estatisticamente semelhantes entre si.

Os dados de rugosidade não apresentaram normalidade. Assim, aplicou-se o teste de Kruskal-Wallis que não demonstrou efeito significativo de tratamento $(\mathrm{P}=0,08)$, não havendo diferença estatística entre as médias de rugosidade para os grupos experimentais. Os valores estão descritos na Tabela 4

Tabela 4 - Resultados para o teste de rugosidade

\begin{tabular}{l|l}
\hline \multicolumn{1}{c|}{ Grupo } & \multicolumn{1}{c}{ Mediana } \\
\hline Z1D & $0,2 \mathrm{~A}$ \\
Z1I & $0,1 \mathrm{~A}$ \\
Z35I & $0,09 \mathrm{~A}$ \\
OD & $0,2 \mathrm{~A}$ \\
Z35D & $0,1 \mathrm{~A}$ \\
OI & $0,1 \mathrm{~A}$ \\
SI & $0,08 \mathrm{~A}$ \\
\hline
\end{tabular}

\section{Discussão}

As resinas compostas, cada vez mais, estão sendo apresentadas aos profissionais como uma opção para tratamento restaurador de acordo com indicações específicas. Todavia, para que determinado material seja aceito e largamente empregado, suas propriedades devem ser clinicamente comprovadas ${ }^{17}$. Os estudos laboratoriais são grandes aliados na compreensão do comportamento e das propriedades desses compósitos ${ }^{18}$. 
Os testes mais frequentemente utilizados para se determinar a dureza dos materiais dentários são conhecidos pelos nomes de: Brinell, Rockwell, Vickers e Knoop. Entretanto, apenas as durezas Vickers e Knoop avaliam microdureza, pois utilizam área pequena do material e pouca profundidade (menor que $19 \mu \mathrm{m}$ ). Já as durezas Brinell e Rockwell, também denominadas macrodurezas, utilizam áreas extensas de um determinado material para análise da dureza. Por tais motivos, as durezas Vickers e Knoop são comumente empregadas para análise de materiais odontológicos, como as resinas compostas $^{13,19}$.

Uma propriedade que influencia diretamente a longevidade clínica de um material resinoso é a sua microdureza, já que existe uma relação direta entre essa propriedade e a resistência ao desgaste desses materiai ${ }^{20}$. As restaurações confeccionadas com resinas compostas que apresentem menor microdureza são supostamente menos resistentes aos esforços mastigatórios e apresentam menor longevidade clínica ${ }^{21,22}$.

As propriedades dos compósitos são influenciadas pelo tamanho, tipo e volume das partículas de carga e pela proporção com que essa carga é aderida à sua matriz resinosa ${ }^{23}$. Alguns autores evidenciaram que quanto maior a quantidade de carga de um compósito maior será a sua microdureza ${ }^{24,25}$. O conhecimento de sua formulação é de grande importância, pois nos possibilita uma estimativa de suas propriedades mecânicas ${ }^{23}$.

Neste estudo, pôde-se verificar que a resina direta $\mathrm{Z} 100^{\circledR}$ apresentou maior valor de microdureza, quando comparada aos grupos testados, já a Solidex exibiu o menor valor. Os grupos Z350 ${ }^{\circledR}$ e Opallis ${ }^{\circledR}$ apresentaram resultados médios para microdureza.

Analisando a quantidade de carga das resinas compostas, é possível observar que a resina com maior quantidade de carga (Z1D e Z1I) foi também a que apresentou maior valor de microdureza. A ocorrência de tais resultados pode, em parte, ser sustentada pela sua composição. A resina composta microhíbrida $\mathrm{Z}_{100^{\circledR}}$ apresenta uma variada distribuição de tamanhos de partículas. Essa grande distribuição pode levar à maior quantidade de partículas de carga e, consequentemente, ao aumento da dureza e resistência final do material ao desgaste. A resina $\mathrm{Z} 100^{\circledR}$ apresenta $71 \%$ do volume de zircônia e sílica, em contrapartida, a resina indireta Solidex apresentou menor quantidade de carga, $53 \%$ do volume de microcarga de cerâmica inorgânica, justificando menor microdureza ${ }^{26}$.

Além da quantidade de carga, o tipo de matriz polimérica influencia o grau de conversão e, assim, as propriedades dos compósitos. As melhores propriedades físicas e mecânicas são relatadas como uma combinação tanto da composição do material como da sua devida polimerização $0^{27}$.
A matriz orgânica da resina $\mathrm{Z} 100^{\circledR}$ consiste basicamente em Bis-GMA e TEGDMA, já a Solidex é constituída por Bis-GMA, UDMA, TEGMA. Os monômeros UDMA (Uretano dimetacrilato) e Bis-EMA (Bisfenol A - polietileno glicol dieter dimetacrilato), presentes também em outros grupos testados, são de alto peso molecular e, consequentemente, têm poucas ligações duplas por unidade de peso, por isso, geram menor quantidade de radicais livres, resultando em menor grau de polimerização. Provavelmente, a Z100 ${ }^{\circledR}(3 \mathrm{M} / \mathrm{ESPE})$ gerou maior número de radicais livres, resultando em maior grau de polimerização, maior microdureza e melhor desempenho mecânico ${ }^{28,29}$.

As resinas indiretas são adaptações das resinas para uso direto com diferenças, principalmente na forma de polimerização, oferecendo uma cura mais completa e melhores propriedades mecânicas. A realização de restaurações indiretas tem sido proposta com o objetivo de minimizar a tensão gerada sobre a zona de união quando da fotoativação do compósito, bem como possibilitar melhor restituição da forma perdida pelo elemento dentário ${ }^{30}$. Apesar de o presente estudo ter verificado menor valor de microdureza para a resina indireta Solidex, estudos adicionais sobre o grau de conversão e demais propriedades mecânicas dos materiais estudados devem ser realizados, uma vez que um teste isolado não pode definir as propriedades do material.

Com relação à avaliação da qualidade da superfície de materiais dentários, o parâmetro frequentemente utilizado é a rugosidade superficial média, ou seja, valores de Ra. Esse parâmetro é definido como o desvio absoluto da média ao longo do comprimento da amostragem, ou seja, é a média aritmética entre os picos e os vales percorridos pelo instrumento. É o mais usado universalmente devido ao controle de qualidade ${ }^{31}$.

O tamanho e a distribuição das partículas da resina composta têm efeito significativo sobre a rugosidade superficial, que é mais rugosa em compósitos que têm partículas de carga inorgânica maiores ${ }^{32}$. Os resultados mostraram que não houve diferença significativa entre os grupos testados quanto à rugosidade. Ao compararmos os resultados obtidos em nosso experimento com outros em que a rugosidade superficial dependente do material e das técnicas, verificamos que todos os compósitos avaliados se enquadram nos parâmetros estabelecidos, que indicam um limiar de rugosidade superficial inferior ao $\mathrm{Ra}=0,2 \mu^{32,33}$. As resinas devem ser altamente polidas, apresentando níveis de rugosidade e porosidade mais baixos possíveis para dificultar a retenção de biofilme dental. 


\section{Conclusão}

De acordo com a análise dos resultados pode-se concluir que:

- a polimerização adicional dos grupos indiretos não foi capaz de aumentar os valores de microdureza;

- a rugosidade enquadra-se no limiar $(\mathrm{Ra}=0,2 \mu \mathrm{m})$ estabelecido por Bollen et al. 1997, sem apresentar diferenças estatísticas entre os materiais analisados;

- o conteúdo de carga inorgânica, a matriz orgânica e o grau de conversão influenciam nas propriedades mecânicas;

- as resinas de uso direto com polimerização adicional podem ser bem utilizadas na forma indireta.

\section{Abstract}

Objective: to assess superficial roughness by mechanical contact profilometry and to assess the resistance to Knoop microhardness in resins light-activated by either conventional means or with systems of additional polymerization. Materials and methods: eighty-two (82) specimens were distributed in the following groups: SI: Solidex ${ }^{T M}$, Z1D: Z100 TM, Z35D: Z350 TM, OD: Opallis ${ }^{T M}$, Z11: Z100 TM, Z35I: Z350TM, and OI: Opallis ${ }^{T M}$. Resin increments were light cured for 40 seconds each. The group containing the resin of indirect use Solidex ${ }^{T M}(S I)$ and groups $\mathrm{Z1I}, \mathrm{Z35I}$, and OI containing resins of direct use assessed as indirect, were light cured for 40 seconds plus 7 additional minutes with EDG-LUX. Samples were stored in distilled water at $37^{\circ} \mathrm{C}$ for 24 hours. Then, the roughness test was performed and, subsequently, the test of superficial microhardness. The means collected were analyzed by the Kruskal-Wallis non-parametric test and Dunn's multiple comparison test. Results: re$\sin Z 100^{\mathrm{TM}}$, recommended for direct use, obtained the highest microhardness values, regardless of additional light curing. Nonetheless, resin Solidex, recommended for indirect use, obtained the lowest microhardness values. The roughness values did not present statistical differences among the materials analyzed. Conclusion: resins of direct use with additional polymerization may be satisfactorily used indirectly.

Keywords: Composite Resins. Microhardness. Roughness.

\section{Referências}

1. Silveira RR, Castro JCO, Pompeu JGF, Brandim AS, Araújo AAVL, Barros GA. Análise comparativa da microdureza superficial e profunda entre uma resina microhíbrida e uma resina composta de nanopartículas. Pesq Bras Odontoped Clin Integr 2012;12(4):529-34.

2. Hammesfahr PD, O'Connor MT, Wang X. Light-curing technology: past, present, and future. Compendium 2003;23:18-24.

3. Hansen EK, Asmussen E. Correlation between depth of cure and temperature rise of a light-activated resin. Scand J Dent Res 1993;101(3):176-9.

4. Bianchi EC, Aguiar PR, Alves MCS, Freitas CA, Rodrigues AR, Júnior OBC. Comparação dos valores de desgaste abrasivo e de microdureza de 13 resinas compostas usadas em odontologia através do método do disco retificado. Polímeros [online] 2007;17(2):130-6.

5. Silva EM, Poskus LT, Guimarães JGA. Influence of light-polymerization modes on the degree of conversion on mechanical properties of resin composites: a comparative analysis between a hybrid and a nanofilled composite. Oper Dent 2008;33(3):287-93.

6. Hosseinalipour M, Javadpour J, Rezaie H, Dadras T, Hayati AN. Investigation of mechanical properties of experimental Bis-GMA/TEGDMA dental composite resins containing various mass fractions of silica nanoparticles. J Prosthodont 2010;19(2):112-7.

7. Andrade MV, Oliveira LGF, Filho PFM, Silva CHV. Tendências das resinas compostas nanoparticuladas. Int J Dent 2009;8(2):102-8

8. Casselli DS, Worschech CC, Paulillo LA, Dias CT. Diametral tensile strength of composite resins submitted to different activation techniques. Braz Oral Res 2006;20(3):214-8.

9. Asmussen E, Peutzfeldt A. Influence of UEDMA, BisGMA and TEGDMA on selected mechanical properties of experimental resin composites. Dent Mater 1998;14(1):51-6.

10. Elossais AA. Avaliação comparativa in vitro da rugosidade superficial de resinas compostas submetidas a diferentes técnicas de polimento [Dissertação de Mestrado]. Araraquara: Faculdade de Odontologia de Araraquara, Universidade Estadual Paulista.

11. Hirata R, Mazzetto AH, Yao E. Alternativas Clínicas de Sistemas de Resinas Compostas Laboratoriais - Quando e Como Usar. JBC, Curitiba-PR 2000;4(19):13-21.

12. Casselli DSM, Faria e Silva AL, Casselli H, Martins LRM. Effect of cavity preparation design on the fracture resistance of directly and indirectly restored premolars. Braz Oral Sciences 2008;7(27):1636-40.

13. Souza ROA, Michida SMA, Zogheib LV. Avaliação da dureza Vickers de resinas compostas de uso direto e indireto. Cienc Odontol Bras 2009;12(1):23-30.

14. Marchan SM, White D, Smith WA, Raman V, Coldero L, Dhuru V. Effect of reduced exposure times on the microhardness of nanocomposites polymerized by QTH and secondgeneration LED curing lights. Oper Dent 2011;36(1):98-103.

15. Hosseinalipour M, Javadpour J, Rezaie H, Dadras T, Hayati NA. Investigation of mechanical properties of experimental Bis-GMA/TEGDMA dental composite resins containing various mass fractions of silica nanoparticles. J Prosthodont 2010;19(2):112-7.

16. Beun S, Glorieux T, Devaoux J, Vreven J, Leloup G. Characterization of nanofilled compared to universal and microfilled composites. Dent Mater 2007;23(1):51-9.

17. Reges RV, Corrêa FOB, Adabo GL, Cruz CAS, Sobrinho LC. Análise quantitativa do conteúdo de carga inorgânica das resinas compostas. Pós Grad Rev Odontol 2002;5(2):18-22.

18. Gouvêa CVD, Couto CF, Moraes CM, Barros RN. Resistência à compressão entre uma resina composta direta $\mathrm{e}$ dois cerômeros. Rev Odonto Ciência - Fac. Odonto/PUCRS 2007;22(56):138-142.

19. Anusavice KJ, Phillips RW. Materiais Dentários. Rio de Janeiro: Elsevier Editora; 2005. 764p.

20. Condon JR, Ferracane JL. In vitro wear of composite with varied cure, filler level, and filler treatment. J Dent Res 1997;76:1405-11.

21. Oberholzer TG, Du Preez IC, Kidd M. Effect of LED curing on the microleakage, shear bond strength and surface hardness of a resin-based composite restoration. Biomaterials 2005;26(18):3981-6.

22. Peris AR, Mitsui FHO, Amaral CM, Ambrosano GMB, Pimenta LA. The effect of composite type on microhardness 
when using quartz-tungsten-halogen (QTH) or LED lights. Oper Dent 2005;30(5):649-54.

23. Miranda CB, Pagani C, Bottino MC, Benetti AR. A comparison of microhardness of indirect composite restorative materials. J Appl Oral Sci 2003;11(2):157-61.

24. McCabe JF. Applied dental materials. 7. ed. Oxford, England: Blackwell Scientific Publications; 1990. p. 78-86.

25. Willems G, Lambrenhes P, Braem M, Celis JP, Vanherle G. A classification of dental composites according to their morphological and mechanical characteristics. Dent Mater 1992;8:310-9.

26. 3M ESPE. Filtek ${ }^{\mathrm{TM}} \mathrm{Z} 350 \mathrm{XT}$ resina composta universal: perfil técnico do produto Filtek ${ }^{\mathrm{TM}}$. 3M do Brasil 2010.

27. Tanoue N, Matsumura H, Atsuta M. Wear and surface roughness of current prosthetic composites after toothbrush/ dentifrice abrasion. J Prosthet Dent 2000;84:93-7.

28. Fontes AS, Mauro ED, Sano W, Lima NCS, Antonia LHD. Avaliação do Comportamento de Radicais Livres e Desempenho Mecânico de Compósitos Restauradores Dentais Comerciais por Ressonância Paramagnética Eletrônica (RPE) Combinada às Análises Convencionais. Polímeros: Ciência e Tecnologia 2009;19(4):285-91.

29. Ottaviani MF, Fiorini A, Mason PN, Corvaja C. Electron Spin Resonance studies of dental composites: effects of irradiation time, decay over time, pulverzation, and temperature variations. Dent Mater 1992;8:118-24.

30. Lambert PL, Bezerra RB. Restaurações inlay/onlay em porcelana: uma revisão de literatura. Rev da Fac Odontol UFBA 2000;20:32-8.

31. Gadelmawla ES, Koura MM, Makoud TMA, Elewa IM, Soliman HH. Roughness parameters. Processing Technology 2002;123(1):133-45

32. Fagundes TC, Barata TJ, Carvalho CA, Franco EB, van Dijken JW, Navarro MF. Clinical evaluation of two packable posterior composites: a five-year follow-up. J Am Dent Assoc 2009;140(4):447-54.

33. Bollen CML, Lambretchts P, Quirynen M. Comparison of surface roughness of oral hard material to the threshold surface roughness for bacterial plaque retention: a review of the literature. Dent. Mater., Copenhagen 1997;13(3):258-69.

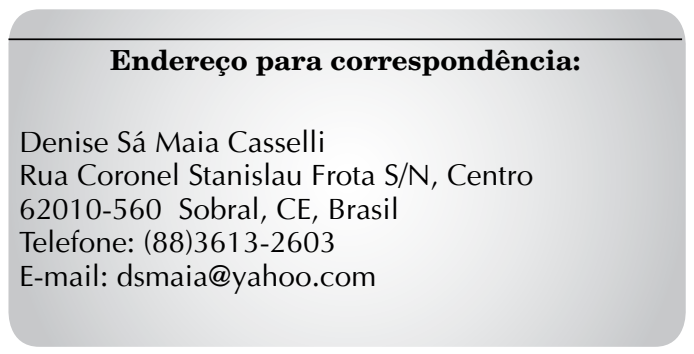

Recebido: 16/10/2014. Aceito: 28/04/2015. 